\title{
In Vitro Evaluation of Anticancer Drugs with Kinetic and Static Alternating Cell Culture System
}

\author{
Yongxin Zhang1 ${ }^{*}$, Ying Wang1, Zhenying Wang1, Farhang Farhangfar ${ }^{2}$, Monica Zimmerman ${ }^{1}$ \\ ${ }^{1}$ Zyxell Inc., Carrollton, TX, USA \\ ${ }^{2}$ Carolinas Healthcare System, Charlotte, NC, USA \\ Email: ^zyx@zyxellinc.com
}

How to cite this paper: Zhang, Y.X., Wang, Y., Wang, Z.Y., Farhangfar, F. and Zimmerman, M. (2017) In Vitro Evaluation of Anticancer Drugs with Kinetic and Static Alternating Cell Culture System. Journal of Cancer Therapy, 8, 845-859.

https://doi.org/10.4236/jct.2017.89074

Received: August 9, 2017

Accepted: September 24, 2017

Published: September 27, 2017

Copyright ( 92017 by authors and Scientific Research Publishing Inc. This work is licensed under the Creative Commons Attribution International License (CC BY 4.0).

http://creativecommons.org/licenses/by/4.0/

\begin{abstract}
Variable bioreactors have been developed for the evaluation of anti-cancer drug efficacy. The Kinetic and Static Alternating Cell Culture System (KSACCS) combines the advantages of kinetic bioreactors and static cultures to improve cell growth by providing adequate metabolic support while minimizing shear-stress. In the current studies, the KSACCS in the ZYX Bioreactor could significantly increase the sensitivity of lung cancer cells (PLS008) and leukemia cells (HL60) to anticancer drugs Cisplatin and 5-FU by accelerating the apoptosis of cancer cells. It was also shown that excessive agitation of the cells could lead to severe cell damage, which resulted in a diminished sensitivity of anticancer drug evaluation, and co-culture systems tend to reduce the sensitivity of anticancer drug evaluation although it might better mimic in vivo conditions.
\end{abstract}

\section{Keywords}

Anticancer Drug Evaluation, Cell Culture, Bioreactor

\section{Introduction}

Rapid and accurate evaluation of anti-cancer drug efficacy is particularly important for anticancer drug screening for cancer patients who will undergo chemotherapy and for the research and development of anticancer medicine. Many efforts have been devoted to the development of in vitro culture systems that more effectively mimic the in vivo environment [1] [2] [3] [4] [5]. Currently, several in vitro culture systems or in vivo animal models are the primary tools used to test cancer cell responses to drugs [3] [4] [5]. However, drug sensitivity data ob- 
tained via two-dimensional (2D) culture systems can be misrepresentative and non-physiologic, while animal models are expensive, time-consuming, and not always predictive of the effects on human tumors in their native environments. Three-dimensional (3D) culture systems, especially with co-cultured supporting cells, which mimic the tumor microenvironment could be a better tool for drug screening by providing a more accurate [6] [7] [8], in vivo-like structure and organization than $2 \mathrm{D}$ culture systems, and without the time and expense associated with the use of animal models [9]-[14]. In addition, culture systems using human tissue or cells as supporting materials may produce responses more predictive of humans than animal models.

A proper representation of the tumor microenvironment is extremely critical for testing the effectiveness of anti-cancer drugs. Since cancer cells in the body exist physically in two main forms, kinetic as in hematopoietic and lymphoid malignancies and static as in solid tumors, an ideal bioreactor system should be able to mimic these two forms in its potential application in anti-cancer agent screening and evaluation. Leukemia cells may spend a majority of their time in peripheral circulation but also reside in the bone marrow. In contrast, most solid tumor cells, such as those in lung and liver cancers, mostly grow in a unique area of the body, save late stage metastatic spread. To mimic these two physical forms in which cancer cells exist in the body, an ideal bioreactor must allow cells to grow well in both static and kinetic states, and these two states should ideally be controlled and regulated in a dynamic fashion by automated control systems. Unfortunately, current commercially-available 3D bioreactors can only maintain cells in either a kinetic state (K bioreactor) [1] [2] [5] [6] [7] or a static state (S bioreactor) [1] [2] [3] [4]. When cells are cultured in a K bioreactor [5] [6] [7], they are kept in motion by agitation or similar mechanism, accumulating at fixed points within the bioreactor when agitation ceases. Cell accumulation adversely affects the growth of cells in culture as cells in the center of the accumulation experience hypoxic conditions and lower concentrations of anti-cancer drugs in the medium. On the other hand, cells in S bioreactors are usually supported by gel-like materials which prevent them from moving as they would in peripheral circulation. Therefore, neither the $\mathrm{K}$ bioreactor nor $\mathrm{S}$ bioreactor meets the requirements for both of the above two physical states.

The ZYX bioreactor system used in this study enables programmed control of many culture parameters. This allows the bioreactor to gently maintain cells in suspension for kinetic cultures and provide an even distribution for static cultures, allowing cells to grow well in both physical states [15]. The advantages of ZYX bioreactor have been well-demonstrated in the culture of stem cells and immunocytes [15] [16]. The current study further confirms the advantages of the ZYX bioreactor by mimicking both kinetic and static states of cancer cell survival in the body to improve the objectivity and accuracy of anti-cancer agent screening and evaluation in vitro.

Solid tumor cells always recruit an organic scaffold in which endothelial cells provide the tumor with vascular support and stromal cells provide the nascent 
tumor with physical support and cytokines, both of which contribute significantly to cancer cell proliferation [14] [17]. Therefore, different cells are required to build an appropriate microenvironment for tumor cell growth. The gradient osmosis perfusion system of the ZYX bioreactor [15] provides the ideal conditions for cell co-culture in our pilot experiment with cancer cells. This study also demonstrated the advantages of the ZYX bioreactor in co-culture by mimicking human tissue and further provided a firm basis for the application of the ZYX bioreactor to potentially increase the accuracy and efficiency of anti-cancer drug evaluation.

\section{Materials and Methods}

\subsection{Characterization of the Advantages of the ZYX Bioreactor Technologies in Anti-Cancer Drug Screening}

Cancer cells used in this project included suspension cell lines: HL-60, and solid cancer cell lines: MCF-7, PC-3, PA-1, and HeLa S3 as well as small cell lung cancer primary cells. Human carcinomas of breast (MCF-7), prostate (PC-3), ovary (PA-1) and cervix (HeLa S3) as well as Leukemia HL-60 were obtained from the American Type Culture Collection. Primary human cancer cells (small cell lung cancer, [PLS008]) [15] were from the ZYX Biotech company. HeLa S3 cells were grown in DMEM. MCF-7 cells were grown in Eagle's MEM supplemented with $10 \mu \mathrm{g} / \mu \mathrm{L}$ insulin. PC-3 cells were grown in Ham's medium/nutrient mixture F-12. PA-1 was grown in DMEM/F-12. HL-60 and PLS008 were cultured in IMDM (ATCC30-2005). All media were supplemented with $10 \%$ fetal bovine serum except for HL-60 cells which were cultured in 20\% FBS. Cells were seeded in $10 \mathrm{ml}$ gas permeable cell culture bags for ZYX bioreactor cultures or conventional static cultures with 6-well plates at the following densities: PLS008 cells at $1 \times 10^{5} / \mathrm{ml}, \mathrm{HeLa}$ and MCF-7 cells at $1 \times 10^{5} / \mathrm{ml}, \mathrm{PC}-3$ cells at $2 \times 10^{4} / \mathrm{ml}$, PA- 1 cells at $2.5 \times 10^{4} / \mathrm{ml}$, and HL-60 cells at $1 \times 10^{5} / \mathrm{ml}$. On culture day 6 , all adherent cells were trypsinized using a standard procedure prior to analysis whereas suspension cells were analyzed directly. All culture media contained 100 $\mathrm{U} / \mathrm{mL}$ penicillin, and $100 \mu \mathrm{g} / \mathrm{mL}$ streptomycin (pH 7.2) (Gibco-BRL, Germany). $10 \mu \mathrm{mol} / \mathrm{L}$ Cisplatin [18] [19] [20] and $10 \mu \mathrm{mol} / \mathrm{L}$ 5-FU [21] [22] [23] [24] were used for the evaluation of anticancer drug sensitivity, and 1.0, 2.5, 5.0 and 10.0 $\mu \mathrm{mol} / \mathrm{L}$ Cisplatin were used for the evaluation of dose-effect relationship.

\subsection{Analysis of the Effects of Agitation on the Evaluation of Anti-Cancer Drugs}

5 different programs (K0, K1, K2, K3 and Kf) of ZYX Bioreactors were used to provide different ratios between static and kinetic states. In these programs, $\mathrm{Kf}$ was a purely kinetic culture setting in which the bioreactor was constantly rotated and K0 provided a static control in which cultures were not rotated during the culture. For the programs K1, K2 and K3, cultures were vertically rotated once, twice, or three times every 24 hours, respectively. Rotation times started 
with 5 minutes each and increased gradually and automatically as the cell expansion fold increased until they reaches Kf level. Small cell lung cancer cells (PLS008) alone and with Cisplatin [18] [19] [20] were used in the tests, and each group was tested four times. At the end of each test, viable cells were assessed by MTT assay and counted by Trypan Blue dye exclusion and flow cytometry (Propidium Iodide [PI] staining for dead cell counting and Annexin V staining for identification of early stage apoptosis as previously described [15] [25]). The ratios of treated/untreated control were used in the statistical analysis for the evaluation of anti-cancer drug efficacy.

\subsection{Establishing Dose-Response Relationships between Cancer Cell Lines and Known Effective Anti-Cancer Agents}

5 different dosages $(0,1,2.5,5$ and $10 \mu \mathrm{mol} / \mathrm{L})$ for Cisplatin and 5FU were applied to PLS008. The efficacy of Cisplatin was evaluated by MTT assay, cell counting and cell apoptosis assessment as described above. In comparison with static culture, a lower dosage of anti-cancer drug or/and a more cancer cell growth inhibition would indicate a higher sensitivity imparted by the ZYX 3D culture system.

\subsection{Establishing a Co-Culture Model to Mimic the Tumor Growth Microenvironment}

Fibroblasts [17] [26] with $6 \mathrm{~mm}$-diameter cell carriers were co-cultured with HL60, in which the media contained human recombinant fibroblast growth factor 9 (FGF9, $10 \mathrm{ng} / \mathrm{ml}$ ) (R \& D Systems, Minneapolis, MI). Two weeks later, suspension cells were harvested and viable cells were assessed as described above. Cultures were grouped based on the application of fibroblasts and the addition of Cisplatin and 5FU. Each group contained 4 cultures.

\subsection{Analysis of the Efficiency, Sensitivity, and Reproducibility of Anti-Cancer Drug Evaluation}

Efficiency was evaluated by measuring the rate of response to the anti-cancer drug (greatest response over the shortest culture incubation time in which anti-cancer drug efficacy could be determined). Sensitivity was evaluated by comparing different culture methods at the same concentration of the drug that showed efficacy. This could also be used to compare the relative effectiveness of various drugs. Reproducibility was evaluated by comparing the coefficients of variation between the cultures with the selected ZYX Bioreactor program and traditional static cultures.

\subsection{Statistics Analysis}

ANOVA and Student t-test were used for comparison of means, including those for cell number and their percentages. Reproducibility was evaluated with coefficient of variations $(\mathrm{CV}=$ standard deviation/mean $\times \%) . P<0.05$ was considered as significant. 


\section{Results}

\subsection{Increased Proliferation of Cancer Cells in the ZYX Bioreactor}

Human breast (MCF-7), prostate (PC-3), ovary (PA-1), cervix (HeLa S3), Leukemia HL-60 and lung cancer cells (PLS008) were cultured at proper seeding densities. On culture day 6, all adherent cells were trypsinized using standard procedure prior to analysis whereas suspension cells were analyzed directly. ZYX Bioreactors with program K2 were compared to six well plates as the static culture controls. Figure 1 shows that all cancer cells grew significantly faster in the ZYX bioreactor than in $2 \mathrm{D}$ static culture $(P<0.01, \mathrm{n}=$ 4/each condition).

\subsection{Increased Effects of Anti-Cancer Drugs to Cancer Cells in the ZYX Bioreactor with Program K2}

PLS008 lung cancer cells and HL-60 Leukemia cells were treated with10 $\mu \mathrm{mol} / \mathrm{L}$ Cisplatin and $10 \mu \mathrm{mol} / \mathrm{L}$ 5FU in a ZYX bioreactor with program K2 (partial kinetic culture) and program k0 (static culture) for 8 days. Cells were collected and counted on days 2, 4, 6 and 8. As shown in Figure 2, PLS008 (Figures 2(a)-2(c)) and HL-60 (Figures 2(d)-(f)) proliferate vigorously in cultures without anti-cancer drugs (Figure 2(a) and Figure 2(d)), with the cells in the partial kinetic cultures (ZYX Bioreactor, program K2) growing faster than those in static cultures (program K0). To clearly exhibit the relative effects of different drugs and physical culture conditions, the no-drug control curves were left out and curves were plotted as percentage change from the seeding density (Figure 2(b)

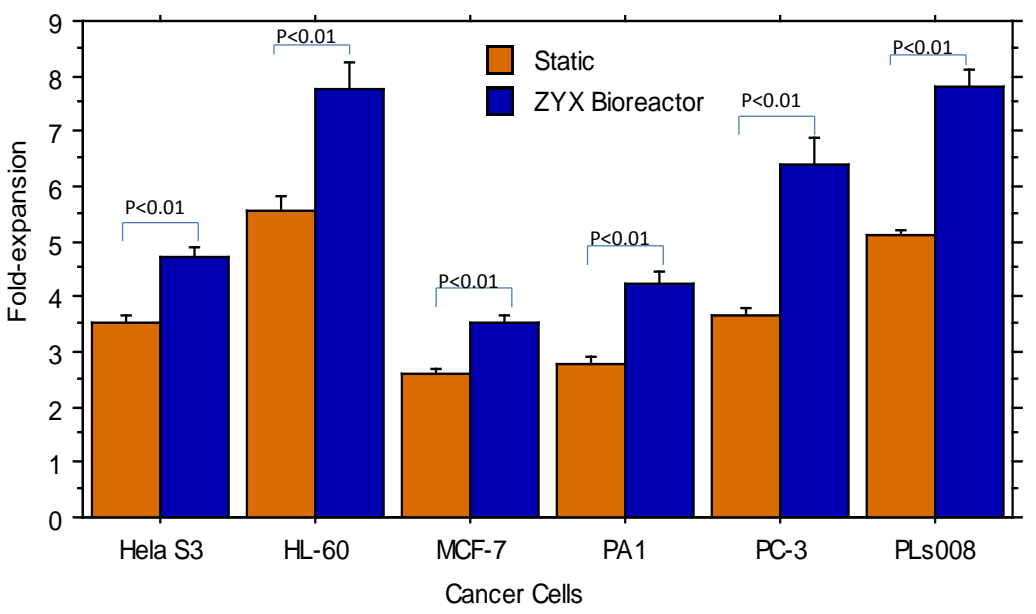

Figure 1. Improved growth of cancer cells in ZYX bioreactor. Human breast (MCF-7), prostate (PC-3), ovary (PA-1), cervix (HeLa S3), Leukemia HL-60 and lung cancer cells (PLS008) were cultured at proper seeding densities, and on culture day 6, all adherent cells were trypsinized using standard procedures prior to analysis whereas suspension cells were analyzed directly. Cultures from ZYX Bioreactors with the program K2 were compared to used as the static culture controls grown in six well plates. Figure 1 shows that all cancer cells grew significantly faster in the ZYX bioreactor than in 2D static culture $(P<0.01, \mathrm{n}=4)$. 


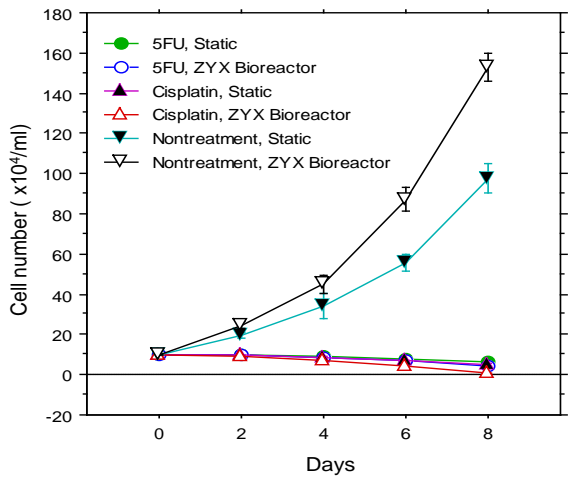

(a)

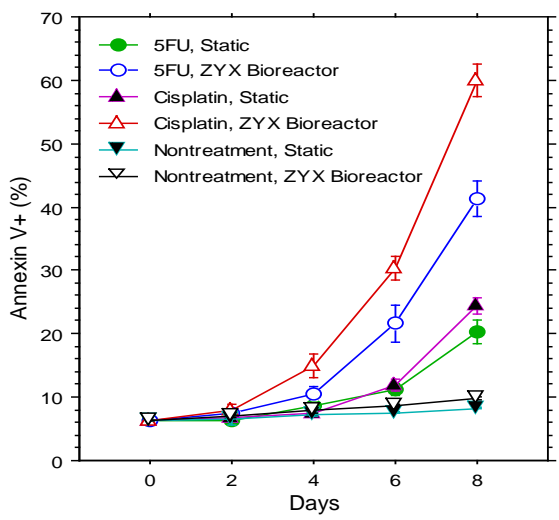

(c)

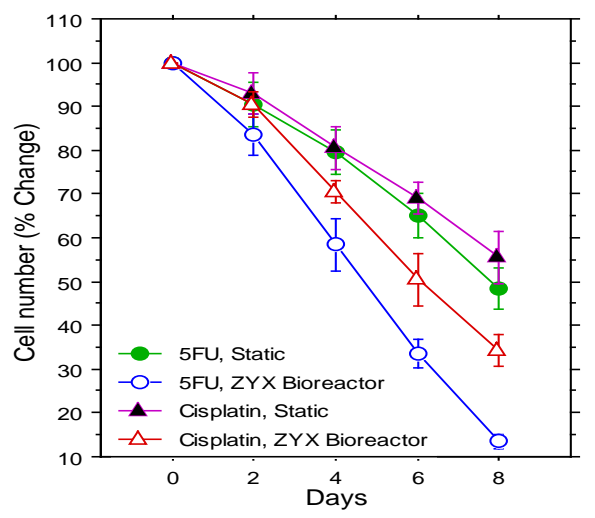

(e)

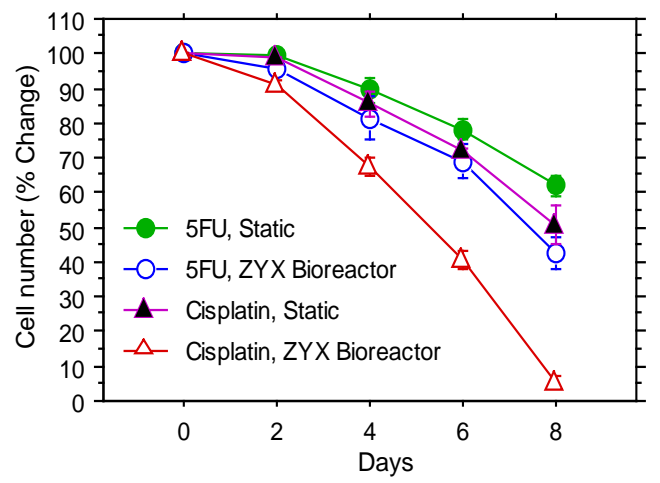

(b)

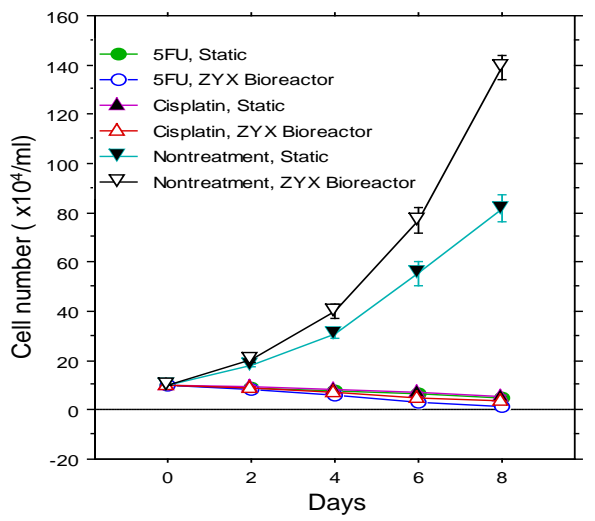

(d)

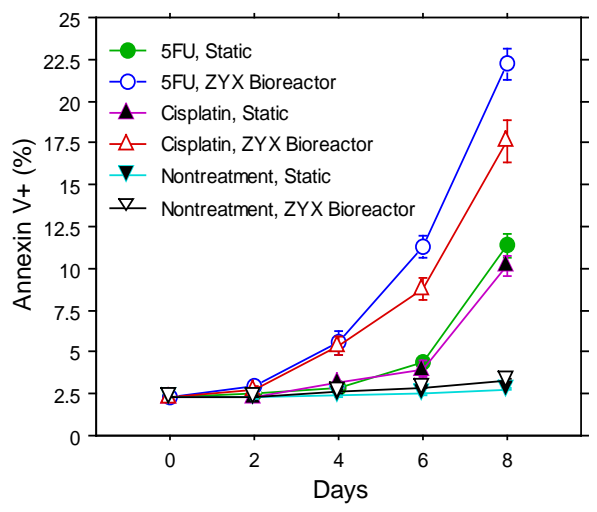

(f)

Figure 2. Increased effects of anti-cancer drugs on cancer cells in the ZYX bioreactor. PLS008 lung cancer cells ((a)-(c)) and HL60 Leukemia cells ((d)-(f)) were treated with $10 \mu \mathrm{mol} / \mathrm{L}$ cisplatin or $10 \mu \mathrm{mol} / \mathrm{L} 5 \mathrm{FU}$ in static cultures and in ZYX bioreactors for 7 days. 24 hours after culture initiation, viable cells were assessed by MTT assay, counted with Trypan Blue dye exclusion and analyzed by flow cytometry (count, PI staining for viability and Annexin V staining for identification of early stage apoptosis). (a), (b), (d) and (e) respectively show changes in cell numbers at collection and cell number percent change (cell density at sample collection time point/cell seeding density x100\%). Cells grew faster ((a) and (d)) and were more sensitive to anticancer drugs in ZYX bioreactor with program K2 ((b) and (e)) compared to static cultures with program K0. The percentage of Annexin V-plus cells drug-treated program K2 increased significantly faster than in static culture $(P<0.05-0.01)$. Both cell types exhibited a significant difference $(P<0.05-0.01)$ in the reduction of cell numbers by Cisplatin compared to $5 \mathrm{FU}$ from day 4 through day $8((\mathrm{~b})$ and (e)) in the cultures with program K2 while Static cultures only demonstrated a significant difference between the effect of the two drugs at day 8. The percentage of Annexin V-positive cells in kinetic cultures increased faster than that of static cultures ((c) and (f)). Annexin $\mathrm{V}$ in the static control cultures without anti-cancer drug only slightly increased with levels significantly lower than the cultures with anticancer drug on day 8. 
and Figure 2(e)). PLS008 cells in ZYX Bioreactors with program K2 were more sensitive to Cisplatin and 5FU than cells in static cultures as shown by the faster reduction of the live cell percentage (Figure 2(b)). HL-60 cells showed a similar trend (Figure 2(e)). The difference between Cisplatin and 5FU in the culture with ZYX Program K2 was significantly greater $(P<0.05-0.01)$ than that in the static culture with program $\mathrm{K} 0$. Annexin $\mathrm{V}$ is a cell marker for early stage apoptosis. The percentage of Annexin V-positive cells in kinetic cultures increased faster than that of static cultures (Figure 2(c) and Figure 2(f)). The difference between Cisplatin and 5FU in the culture with ZYX bioreactor program K2 was also significantly greater than static cultures from day 4 to day 8 . Annexin $\mathrm{V}$ in the control cultures without anti-cancer drug only slightly increased with levels significantly lower than the cultures with anticancer drug on day 8 . The percentage of PI-positive cells was lower than Annexin V-positive cells (45.8\% - 53.6\% less) but showed the same trends as Annexin V (data not shown).

\subsection{Dose-Effect Relationship of Anticancer Drugs in ZYX Bioreactor Cultures}

Lung cancer cells PLS008 were used for the evaluation of dose-effect relationship in responses to cisplatin and 5FU. As the dose (concentration) of anticancer drug increased, the lung cancer cell number significantly reduced, and the cell number change was negatively correlated $(P<0.01, \mathrm{R}=-0.82)$ to the anticancer dose (concentration). When the data was analyzed in semi-Log, the curves were more linearized. Increasing drug dose appeared to cause a logarithmic drop in cell numbers (Figure 3(b)). The significant difference between Cisplatin and $5 \mathrm{FU}$ can be seen at $1 \mu \mathrm{mol} / \mathrm{L}$ in the culture with ZYX bioreactor program $\mathrm{K} 2$ but such a difference can be seen on day 6 in the static culture (program K0).

\subsection{Effect of Different ZYX Bioreactor Programs on the Sensitivity of Anticancer Drugs to Cancer Cells}

ZYX bioreactor program $\mathrm{K} 2$ is favorable for testing sensitivity to anticancer drugs. To further confirm if this advantage is unique to this program, five different ZYX bioreactor programs were used to examine the effects of anticancer drugs $(10 \mu \mathrm{mol} / \mathrm{L}$ each) on cultured cell numbers. The kinetic portion of cultures gradually increased from completely static (K0) to completely kinetic (Kf). As shown in Figure 4, PLS008 lung cancer cells (Figure 4(a) and Figure 4(b)) and HL60 leukemia cells (Figure 4(c) and Figure 4(d)) proliferated significantly more under program $\mathrm{K} 2$ than they did under program $\mathrm{K} 0(P<0.01)$ in the culture without anticancer drugs (non-treatment). Unexpectedly, the growth of both cells sharply decreased from program K2 to Kf $(P<0.01)$, suggesting that kinetic program $\mathrm{K} 2$ provides the most suitable culture condition for the proliferation of these two types of cells.

5FU displayed a greater effect on culture cell numbers than Cisplatin under programs $\mathrm{K} 1, \mathrm{~K} 2$, and $\mathrm{K} 3$ but not $\mathrm{K} 0$ and $\mathrm{Kf}$. This difference was more clearly 


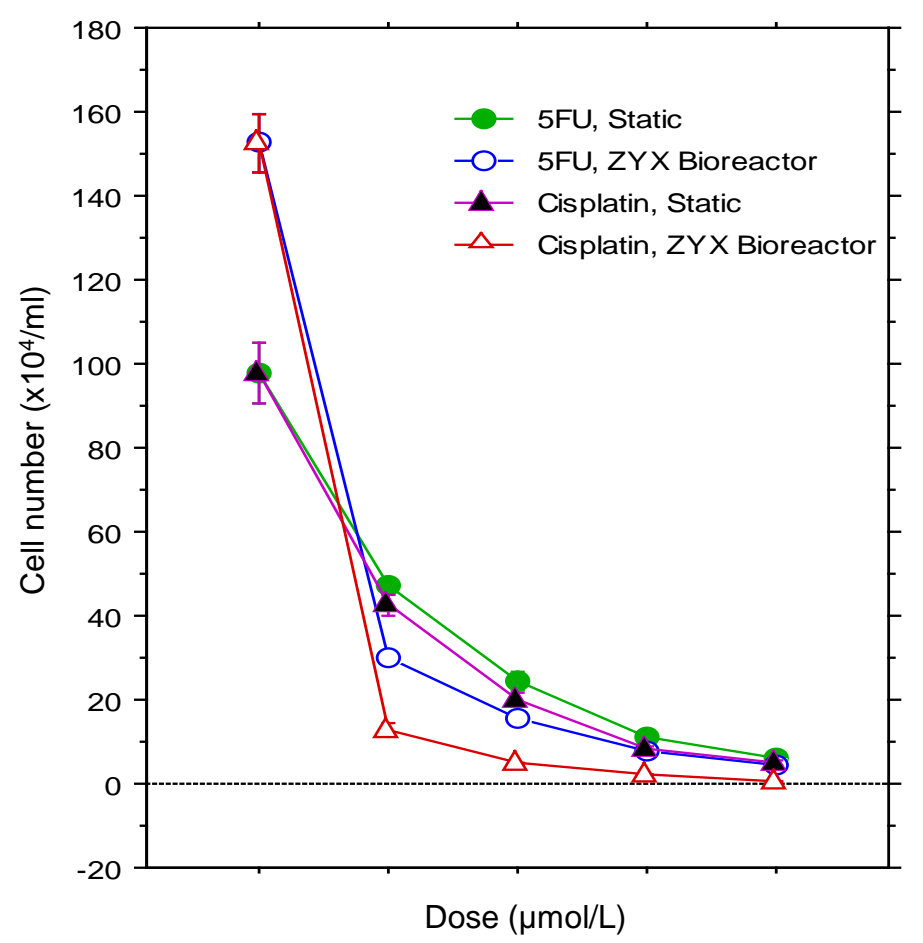

(a)

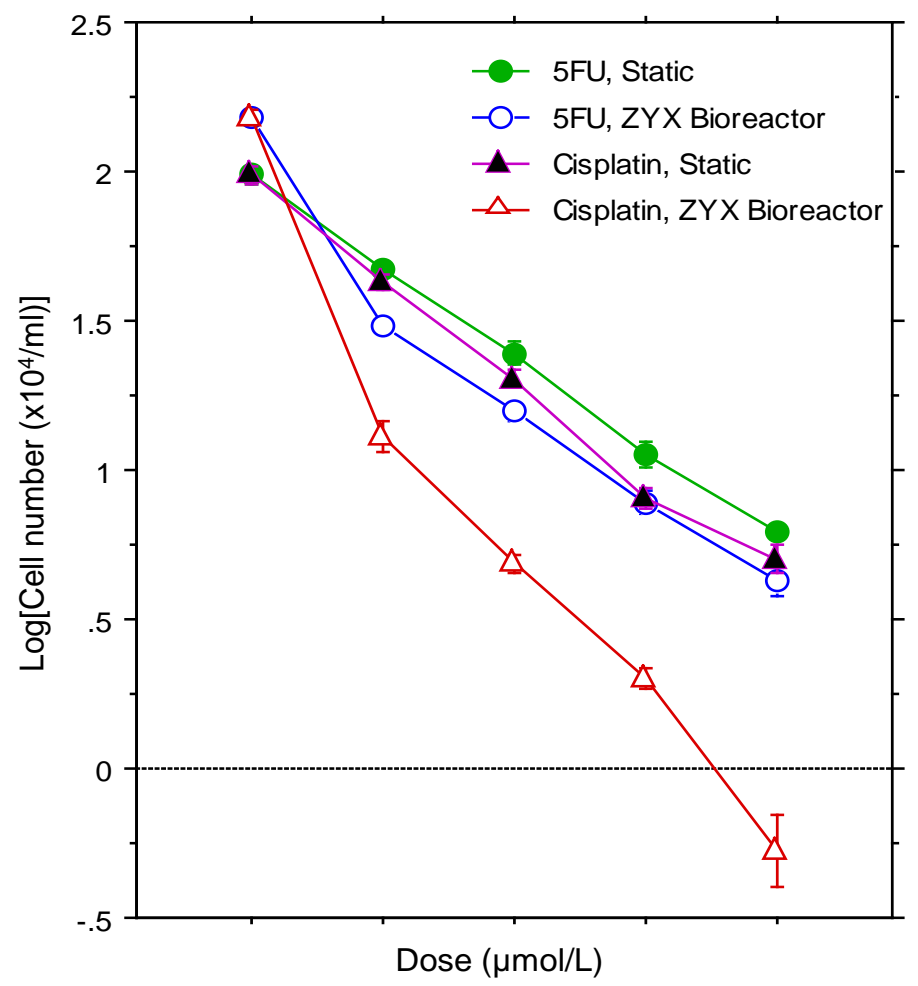

(b)

Figure 3. Dose-effect relationship of Cisplatin and 5FU in lung cancer cell PLS008. PLS008 cells were cultured with $0,1,2.5,5$ and $10 \mu \mathrm{mol} / \mathrm{L}$ Cisplatin or 5FU for 8 days, the dose-effect curves were plotted directly (a) or in semi-Log (b). Compared to (a), the dose-effect curves in (b) are more linearized and show PLS008 was more sensitive to the increasing concentrations of both Cisplatin and 5-FU $(P<0.05-0.01)$. 


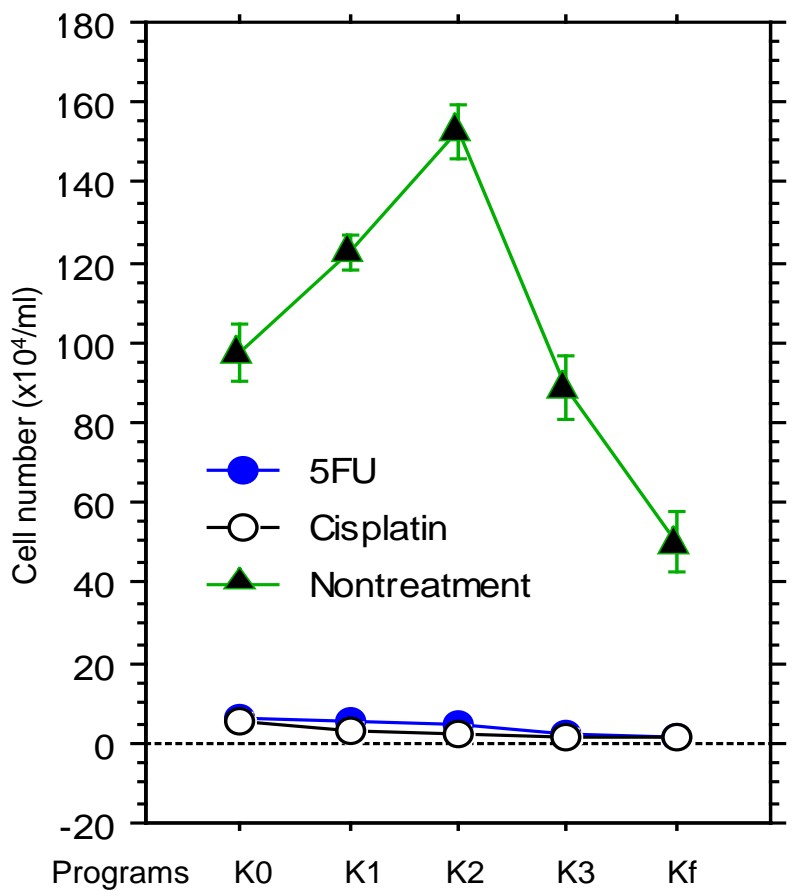

(a)

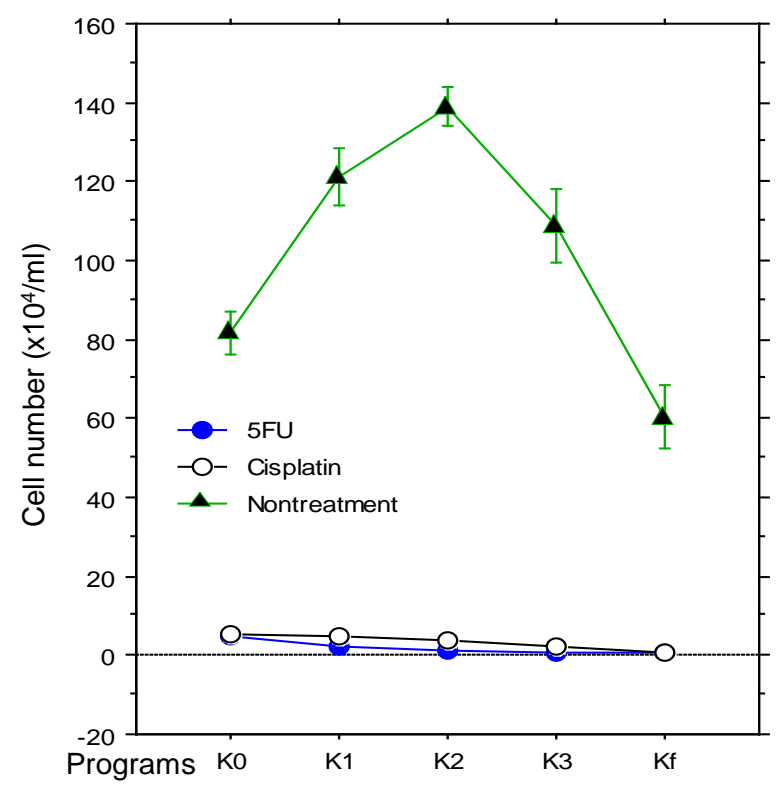

(c)

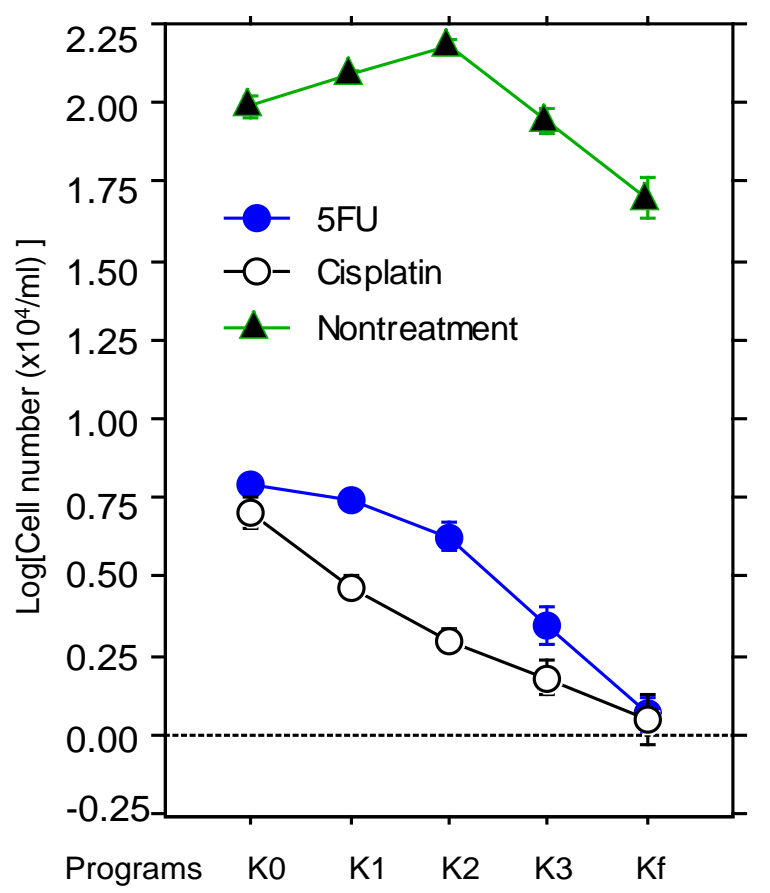

(b)

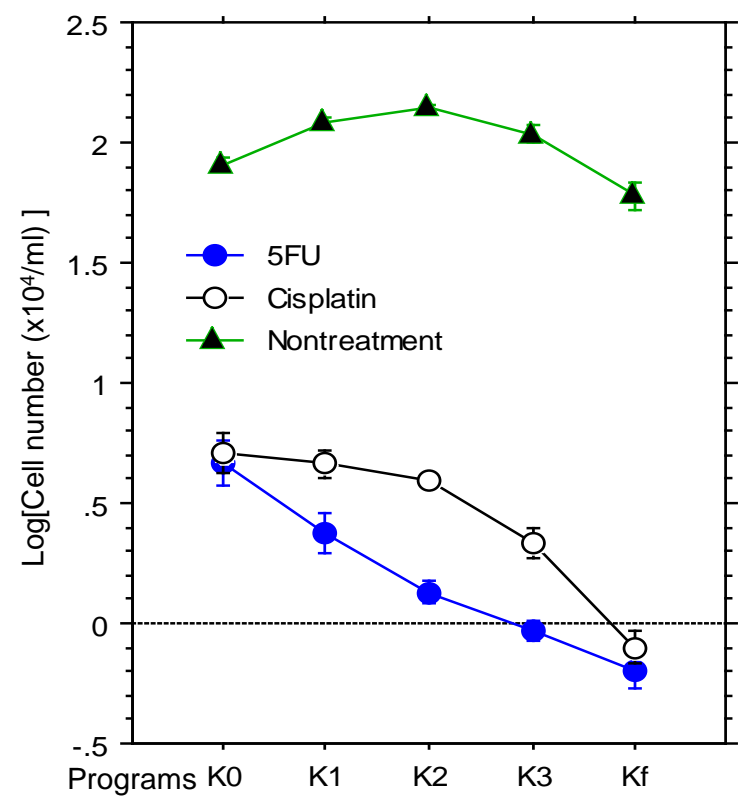

(d)

Figure 4. Effects of different Bioreactor programs on the sensitivity of cancer cells to anticancer drugs. Five different ZYX bioreactor programs were used for the culture of PLS008 and HL-60 cells with Cisplatin, 5FU or a no-drug control. Program K0 is for a static culture, $\mathrm{K} 1$ is for 1 unit ( 5 minutes, once per day) of kinetic culture per day and increases the length of the kinetic culture unit with increasing cell density, $\mathrm{K} 2$ is for 2-units ( 5 minutes, twice per day) of kinetic culture per day and increases the length of the kinetic culture unit with increasing cell density, $\mathrm{K} 3$ is for 3-unit ( 5 minutes, 3 times per day) kinetic culture per day and increases the length of the kinetic culture unit with increasing cell density, and $\mathrm{Kf}$ is for a completely kinetic culture. For the culture without anti-cancer drugs, cell densities increased as the kinetic portion of the culture did, starting with K0 and reaching a peak level with program K2. From that point densities declined rapidly ((a)-(c)). The differences between adjacent programs are significant $(P<0.01)$. Partial kinetic cultures $(\mathrm{K} 1, \mathrm{~K} 2$, and K3) also exhibited significant differences between Cisplatin and 5FU $(P<$ $0.05-0.01$ ), but purely static (K0) and purely kinetic cultures (Kf) did not ((b) and (d)). 
exhibited when the data was plotted in semi-Log format (Figure 4(b) and Figure 4(d)). Flow cytometric analysis revealed similar levels of early apoptotic cells (13.6\% - 25.3\% Annexin V-positive) and dead cells (7.9\% - 14.8\% PI-positive) for day 4 culture with programs $\mathrm{K} 0, \mathrm{~K} 1$ and $\mathrm{K} 2$, but significantly higher ratios with program K3 $(38.3 \%$ - 49.6\% Annexin V-positive and 29.7\% - 45.2\% PI-positive) and Kf (62.5\% - 78.3\% Annexin V-positive and 58.1\% - 65.9\% PI-positive). K0, K1 and K2 day 4 cultures without anticancer drugs had relatively few apoptotic and dead cells (2.9\% - 5.3\% Annexin V-positive and 2.3\% - 4.8\% PI-positive). These levels greatly increased under programs K3 (19.8\% - 25.6\% Annexin V-positive and 8.9\% - 14.8\% PI-positive) and Kf (42.5 - 57.3 Annexin V-positive and $24.7 \%$ - 34.2\% PI-positive).

\subsection{Decreased Sensitivity to Anticancer Drugs in HL60 Cells Co-Cultured with Fibroblasts}

Co-culturing cancer cells with supporting cells provides them with a test environment more similar to in-vivo conditions. Fibroblast support cell cultures were grown to $80 \%$ confluence. At that point, all media was removed and HL60 cells were added in media containing either $5 \mathrm{FU}$ or Cisplatin. Figure 5 shows that co-cultured HL60 cells were significantly less sensitive to both 5FU and Cisplatin than cultures without supporting cells from day 4 to day 8 . Moreover, there was a significant differences between cell numbers in Cisplatin and 5FU cultures without supporting cells on days 4, 6 and 8, but this difference can only seen on the days 6 and 8 in the co-cultured cells.

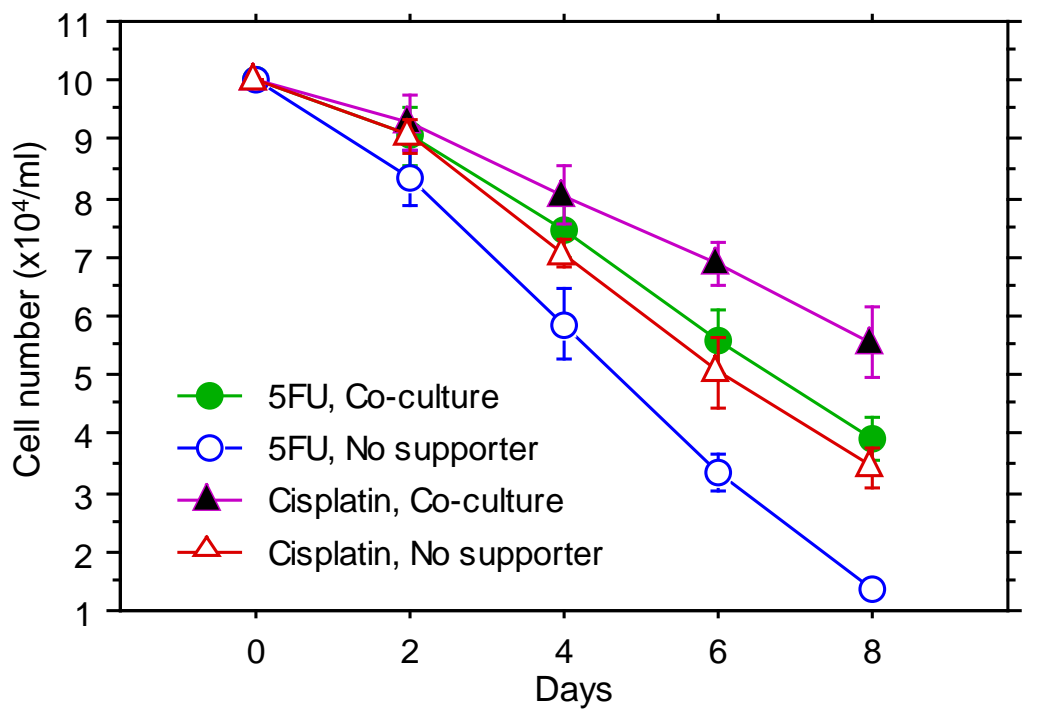

Figure 5. Effects of supporting cells on the sensitivity of cancer cells to anticancer drugs. HL60 cells cultured in media containing anti-cancer drugs Cisplatin and 5FU exhibited a decline in cell density over the course of the culture. Cultures with both drugs exhibited a significantly smaller decline from day 4 to day 8 when the cells were seeded with $80 \%$ confluent fibroblasts $(P<0.05-0.01)$. A significant difference could be observed between Cisplatin and 5FU in the co-cultures at day 6 and day 8 , and in the culture without supporting fibroblasts at day 4 through day 8 . 


\subsection{Evaluation of the Reproducibility of ZYX Bioreactor Programmed Cell Culture in Testing the Sensitivity of Anticancer Drugs}

The coefficients of variation (CV) from 56 pairs of tests (each test has 4 observations, total 448 observations) with the static culture and ZYX Bioreactor programmed cultures were compared and the difference of CVs between these two culture methods was not significant $(P>0.5)$, indicating that the reproducibility of ZYX Bioreactor programmed cell culture is similar to static culture (Figure 6).

\section{Discussion}

Most anti-cancer drugs works more efficiently on faster proliferating cancer cells and cancer cells can grow faster under more suitable culture conditions. Thus, improving the cell growth conditions could make the evaluation of drug efficacy more efficient.

In this study, we showed that culture conditions with alternating kinetic and static phases could be more favorable for cancer cell growth. ZYX bioreactor program K2 provided the most favorable conditions tested for the lung cancer cell line PLS008 and the leukemia cell line HL60. Four other cell lines were also cultured using this program in comparison with conventional static cultures and all showed improved proliferation under program K2.

Cisplatin is clinically used for the chemotherapy of small cell lung cancer [18] [19] [20] and 5FU was approved by the FDA for the treatment of leukemia [21] [22] [23] [24] [26]. These two anti-cancer drugs exhibited clear cytotoxicity in cultures of either the PLS008 lung cancer cell line or the HL60 leukemia cell line. From the data in Figure 2, it can be seen that Cisplatin is more toxic to PLs008 and 5FU is more toxic to HL60, and the cells cultured in the ZYX bioreactor with the program K2 responded to anti-cancer drugs 4 days earlier and more intensely (greater decrease in live cell numbers and higher proportion of cells entering apoptosis). Moreover cultures grown with the program K2 could be used to effectively differentiate between the efficacy of Cisplatin vs. 5FU significantly better than cultures grown under static conditions. These data suggested that kinetic/static alternating cultures could reduce test time and amplify the sensitivity of the evaluation of anti-cancer drug efficacy. Figure 2 also showed that the anti-cancer effects of Cisplatin and 5FU could be seen in 48 hours (Figure 2(a) and Figure 2(d)) and that significant differences between Cisplatin and $5 \mathrm{FU}$ could be seen in less than 4 days in $\mathrm{K} 2$ cultures but this difference was not apparent earlier than day 8 with static cultures (Figure 2(b), Figure 2(c), Figure $2(\mathrm{e})$ and Figure 2(f)).

The induction of cancer cell apoptosis is one of the mechanisms of action anti-cancer drugs [27]-[32]. The current study demonstrates that the proper ZYX bioreactor program can enhance the effects of anti-cancer drugs on cancer cells by increasing apoptosis, as shown by a more rapidly increase in Annexin $\mathrm{V}$-positive and PI-positive cells. 
The window for observing the dose-effect relationship between anti-cancer drugs and in vitro cultured cancer cells is relatively narrow since the toxicity of the drug often leads the cells death in the early stages of the culture. For this reason, most of the tests in the current study were limited to 8 days. As shown in Figure 3 , even at $1 \mu \mathrm{mol} / \mathrm{L}$ of Cisplatin or $5 \mathrm{FU}$ caused a sharp drop in the number of live lung cancer cells. The two drugs had significantly different efficacy which was clearly exhibited at all tested doses in cultures grown in the ZYX bioreactor with program K2 but not in static cultures (Figure 3(b)).

ZYX Bioreactor is a programmable automatic cell culture system with many internal programs. These programs can determine various culture parameters including the kinetic/static status. To test if increasing the kinetic portion of the culture could further elevate the efficiency of the evaluation of anti-cancer drugs, 5 different programs were selected. These ranged from completely static (K0) to completely kinetic (Kf) and were applied in the experiment as shown in Figure 4. The program $\mathrm{K} 2$ was found to be optimal in non-treatment control cultures as described above. This program was also best at differentiating between the effectiveness of Cisplatin and 5FU on cancer cell cultures (Figure 4(b) and Figure $4(\mathrm{~d})$ ), suggesting that the ZYX bioreactor can be used to optimize culture conditions and improve the sensitivity of such drug evaluations.

Flow cytometric analysis revealed that apoptotic Annexin V and PI-positive cells greatly increased in cancer cell cultures with program $\mathrm{K} 3$, which has a greater kinetic portion, and $\mathrm{Kf}$, which is a purely kinetic program. This effect was evident in both untreated cultures and anti-cancer drug treated cultures. This is consistent with earlier findings which reported that ceaseless kinetic cultures resulted in damage to cell membranes and organelles and stimulated the nonspecific differentiation of stem cells due to the continuous shear-stress on the cells [7] [15]. Therefore, it is possible that the decreased sensitivity of anti-cancer drug evaluation and decline in untreated cell proliferation in cultures with a greater kinetic portion resulted from cell damage caused by excessive shear-stress.

Co-cultures with human tissues or human cells provide the cells with a more in-vivo-like growth environment, and may more reliably reflect bona fide effects of anti-cancer drug on cancer cells [12] [17] [20]. HL-60 is a leukemia cell line, which was originally supported by fibroblasts and mesenchymal cells in bone marrow. These supporting cells are biologically similar. When HL-60 cells were co-cultured with fibroblasts, the toxicity of anti-cancer drugs to the cancer cells was reduced and the sensitivity of the test was decreased, although co-cultured cells grown using the ZYX bioreactor program K2 might still perform better than static mono-cultures and could differentiate between Cisplatin and 5FU in 6 days compared to 8 days for static cultures (Figure 2 and Figure 5). Larger dose of drug or extended cultures may improve the effectiveness of anti-cancer drug test in co-cultures.

Coefficient of variation is often used to assess repeatability and reproducibility when replicate measurements by each method are available [33] [34]. 


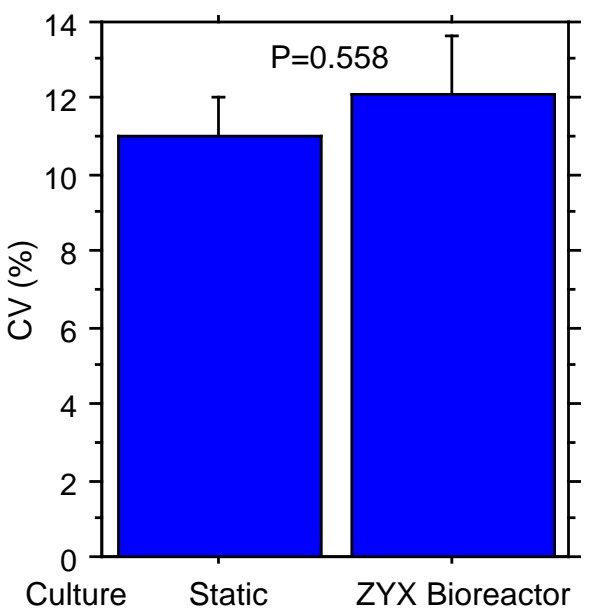

Figure 6. Comparison of reproducibility between static culture (K0) and the culture with peak ZYX Bioreactor program (K2). The coefficients of variation (CV) from 56 pairs of tests (each test has 4 observations) with the culture program K0 and K2 were compared and the difference between these two culture methods was not significant $(P>0.5)$.

Although the sensitivity of anticancer drug evaluation in cultures with the kinetic and static alternating program $\mathrm{K} 2$ in the ZYX Bioreactor is significantly greater than that of static cultures, these two methods exhibit similar mean coefficients of variation, suggestive of reliable reproducibility of tests comparing these culture systems.

\section{Conclusion}

Kinetic and static alternating cell culture could significantly increase the sensitivity of cancer cells to anticancer drugs, in which the accelerated apoptosis of cancer cells were involved. The excessive agitation of the cells could lead to severe cell damage, which resulted in a diminished sensitivity of anticancer drug evaluation. The co-culture systems might better mimic in vivo conditions but tend to reduce the sensitivity of anticancer drug evaluation.

\section{References}

[1] Haycock, J.W. (2011) 3D Cell Culture: A Review of current Approaches and Techniques. Methods in Molecular Biology, 695, 1-15. https://doi.org/10.1007/978-1-60761-984-0_1

[2] Goral, V.N., et al. (2014) Microstructured Multi-Well Plate for Three-Dimensional Packed Cell Seeding and Hepatocyte Cell Culture. Biomicrofluidics, 8, 046502. https://doi.org/10.1063/1.4892978

[3] Justice, B.A., et al. (2009) 3D Cell Culture Opens New Dimensions in Cell-Based Assays. Drug Discovery Today, 14, 102-107.

[4] Huh, D., et al. (2011) Reconstituting Organ-Level Lung Functions on a Chip. Trends in Cell Biology, 21, 745-754. https://doi.org/10.1016/j.tcb.2011.09.005

[5] Margolis, L., et al. (1999) Long Term Organ Culture of Human Prostate Tissue in a NASA Designed Rotating Wall Bioreactor. Journal of Urology, 161, 290-297. https://doi.org/10.1016/S0022-5347(01)62134-7 
[6] Radtke, A.L., et al. (2012) Culturing and Applications of Rotating Wall Vessel Bioreactor Derived 3d Epithelial Cell Models. Journal of Visualized Experiments, 62, e3868.

[7] Begley, C., et al. (2000) The Fluid Dynamic and Shear Environment in the NASA/JSC Rotating-Wall Perfused-Vessel Bioreactor. Biotechnology and Bioengineering, 70, 32-40. https://doi.org/10.1002/1097-0290(20001005)70:1<32::AID-BIT5>3.0.CO;2-V

[8] Lee, E.J., et al. (2010) Layer by Layer Three-Dimensional Tissue Epitaxy by Cell-Laden Hydrogel. Tissue Engineering Part C: Methods, 16, 157-166. https://doi.org/10.1089/ten.tec.2009.0179

[9] Chitcholtan, K., et al. (2013) Differences in Growth Properties of Endometrial Cancer in Three Dimensional (3D) Culture and 2D Cell Monolayer. Experimental Cell Research, 319, 75-87.

[10] Chandrasekaran, S., et al. (2012) Effect of Homotypic and Heterotypic Interaction in 3D on the E-Selectin Mediated Adhesive Properties of Breast Cancer Cell Lines. Biomaterial, 33, 9037-9048. https://doi.org/10.1016/j.biomaterials.2012.08.052

[11] Martin, K.J., et al. (2008) Prognostic Breast Cancer Signature Identified from 3D Culture Model Accurately Predicts Clinical Outcome across Independent Datasets. PLoS ONE, 3, e2994. https://doi.org/10.1371/journal.pone.0002994

[12] Kenny, H.A., et al. (2007) Use of a Novel 3D Culture Model to Elucidate the Role of Mesothelial Cells, Fibroblasts and Extra-Cellular Matrices on Adhesion and Invasion of Ovarian Cancer Cells to the Omentum. International Journal of Cancer, 121, 1463. https://doi.org/10.1002/ijc.22874

[13] Kenny, P.A., et al. (2007) The Morphologies of Breast Cancer Cell Lines in Three-Dimensional Assays Correlate with Their Profiles of Gene Expression. Molecular Oncology, 1, 84.

[14] Fischbach, C., et al. (2009) Cancer Cell Angiogenic Capability Is Regulated by 3D Culture and Integrin Engagement. PNAS, 106, 399.

https://doi.org/10.1073/pnas.0808932106

[15] Zhang, Y., Wang, Y., Wang, Z., Zhang, M. and Wang, Z. (2016) Cancer Specific CTL Expansion with ZYX Bioreactor. Journal of Clinical \& Cellular Immunology, 7, 398. https://doi.org/10.4172/2155-9899.1000398

[16] Zhang, Y., Wang, Y., Zhang, M., Liu, L. and Mbawuike, I.N. (2016) Restoration of Retarded Influenza Virus-Specific Immunoglobulin Class Switch in Aged Mice. Journal of Clinical and Cellular Immunology, 7, 403.

[17] Yamazoe, H., Hagihara, Y. and Kobayashi, H. (2016) Multicomponent Coculture System of Cancer Cells and Two Types of Stromal Cells for in Vitro Evaluation of Anticancer Drugs. Tissue Engineering Part C: Methods, 22, 20-29. https://doi.org/10.1089/ten.tec.2015.0188

[18] Barr, M.P., et al. (2013) Generation and Characterisation of Cisplatin-Resistant Non-Small Cell Lung Cancer Cell Lines Displaying a Stem-Like Signature. PLoS ONE, 8, e54193. https://doi.org/10.1371/journal.pone.0054193

[19] Shen, D.W., et al. (1995) Characterization of High-Level Cisplatin-Resistant Cell Lines Established from a Human Hepatoma Cell Line and Human KB Adenocarcinoma Cells: Cross-Resistance and Protein Changes. British Journal of Cancer, 71, 676. https://doi.org/10.1038/bjc.1995.134

[20] Schreiber-Brynzak, E., et al. (2015) Three-Dimensional and Co-Culture Models for Preclinical Evaluation of Metal-Based Anticancer Drugs. Invest New Drugs, 33, 835-847. https://doi.org/10.1007/s10637-015-0260-4 
[21] Yang, C.Z., et al. (1995) Multidrug Resistance in Leukemic Cell Line K562/A02 Induced by Doxorubicin. Acta Pharmacologica Sinica, 16, 333.

[22] Chu, E., et al. (1990) Interaction of Gamma Interferon and 5-Fluorouracil in the H630 Human Colon Carcinoma Cell Line. Cancer Research, 50, 5834.

[23] Johnston, P.G., et al. (1992) Immunological Quantitation of Thymidylate Synthase using the Monoclonal Antibody TS 106 in 5-Fluorouracil-Sensitive and -Resistant Human Cancer Cell Lines. Cancer Research, 52, 4306.

[24] Angelis, P.M.D., et al. (2006) Cellular Response to 5-Fluorouracil (5-FU) in 5-FU-Resistant Colon Cancer Cell Lines during Treatment and Recovery. Molecular Cancer, 2, 20. https://doi.org/10.1186/1476-4598-5-20

[25] Zhang, Y., et al. (2002) Apoptosis and Reduced Influenza a Virus Specific CD8+ T Cells in Aging Mice. Cell Death and Differentiation, 9, 651. https://doi.org/10.1038/sj.cdd.4401011

[26] Wang, Z., Wang, Y., Farhangfar, F., Zimmer, M. and Zhang, Y. (2012) Enhanced Keratinocyte Proliferation and Migration in Co-Culture with Fibroblasts. PLOS ONE, 7, e40951. https://doi.org/10.1371/journal.pone.0040951

[27] Kontos, C.K., Christodoulou, M.I. and Scorilas, A. (2014) Apoptosis-Related BCL2-Family Members: Key Players in Chemotherapy. Anti-Cancer Agents in Medicinal Chemistry, 14, 353-374. https://doi.org/10.2174/18715206113139990091

[28] Haq, R., et al. (2013) BCL2A1 Is a Lineage-Specific Antiapoptotic Melanoma Oncogene That Confers Resistance to BRAF Inhibition. Proceedings of the National Academy of Sciences, 110, 4321. https://doi.org/10.1073/pnas.1205575110

[29] Kunkalla, K., et al. (2013) Functional Inhibition of BCL2 Is Needed to Increase the Susceptibility to Apoptosis to SMO Inhibitors in Diffuse Large B-Cell Lymphoma of Germinal Center Subtype. Annals of Hematology, 92, 777. https://doi.org/10.1007/s00277-013-1684-6

[30] Dutta, C., et al. (2012) BCL2 Suppresses PARP1 Function and Nonapoptotic Cell Death. Cancer Research, 72, 4193. https://doi.org/10.1158/0008-5472.CAN-11-4204

[31] Moore, M.J., et al. (2010) An Alternative Splicing Network Links Cell-Cycle Control to Apoptosis. Cell, 142, 625.

[32] Goff, D.J., et al. (2013) A Pan-BCL2 Inhibitor Renders Bone-Marrow-Resident Human Leukemia Stem Cells Sensitive to Tyrosine Kinase Inhibition. Cell Stem Cell, 12, 316.

[33] Hollis, B., et al. (2001) Reproducibility and Repeatability of Transabdominal Uterine Artery Doppler Velocimetry between 10 and 14 Weeks of Gestation. Ultrasound in Obstetrics \& Gynecology, 18, 593-597.

https://doi.org/10.1046/j.0960-7692.2001.00544.x

[34] Antonio, P. (2005) Repeatability and Reproducibility of Fast Macular Thickness Mapping. Archives of Ophthalmology, 123, 1330-1337.

https://doi.org/10.1001/archopht.123.10.1330 
Submit or recommend next manuscript to SCIRP and we will provide best service for you:

Accepting pre-submission inquiries through Email, Facebook, LinkedIn, Twitter, etc. A wide selection of journals (inclusive of 9 subjects, more than 200 journals)

Providing 24-hour high-quality service

User-friendly online submission system

Fair and swift peer-review system

Efficient typesetting and proofreading procedure

Display of the result of downloads and visits, as well as the number of cited articles Maximum dissemination of your research work

Submit your manuscript at: http://papersubmission.scirp.org/

Or contact jet@scirp.org 